\title{
Intermittent nulls in PSR B0818-13, and the subpulse-drift alias mode
}

\author{
G. H. Janssen and J. van Leeuwen
}

\author{
Astronomical Institute, Utrecht University, PO Box 80 000, 3508 TA Utrecht, The Netherlands \\ e-mail: [g.h.janssen; a.g.j.vanleeuwen] @astro.uu.nl \\ Received 8 April 2004 / Accepted 14 June 2004
}

\begin{abstract}
We show that all long nulls in PSR B0818-13 are trains of rapidly alternating nulls and pulses (each shorter than one pulse period). Sometimes only the nulls coincide with our pulse window, resulting in one of the apparently long nulls seen occasionally. We show these are seen as often as expected if during such a train the probability for nulls is 1.2 times less than for pulses.

During nulls, the subpulse drift-speed appears to increase. We assume that the carousel of sparks that possibly underlies the subpulses actually slows down, as it does in similar pulsars like PSR B0809+74, and conclude that the subpulse-drift in this pulsar must be aliased. The carousel must then rotate in $30 \mathrm{~s}$ or less, making it the fastest found to date.
\end{abstract}

Key words. stars: neutron - stars: pulsars: general - stars: pulsars: individual: PSR B0818-13

\section{Introduction}

Observations of drifting subpulses are a nice example of undersampling: in some radio pulsars the positions of the pulse components change regularly (as in Fig. 1), but as these positions are determined only once per pulse period, the exact underlying motion remains unknown.

Finding this underlying motion, and its relation to other pulsar parameters, could be helpful in determining which, if any, of the proposed mechanisms to generate drifting subpulses (Ruderman \& Sutherland 1975; Wright 2003) is correct. We will assume the subpulses are formed by discrete locations of emission ("sparks") that rotate around the magnetic pole of the pulsar, causing the pulse components to move through the pulse windows ("drifting subpulses"). Different directions and speeds of this rotation can produce identical subpulse positions at the observer, so-called aliasing (see also Fig. 2 in van Leeuwen et al. 2003). To determine the actual speed of the spark carousel, one can check for the periodicity caused as brighter or offset sparks re-appear after one carousel rotation. Deshpande \& Rankin (1999) did so for PSR B0943+10, and found a carousel rotation time of around $41 \mathrm{~s}$, longer than expected from theory (Ruderman \& Sutherland 1975). With the rotation time known, Deshpande \& Rankin (1999) could track individual sparks. They noted that sparks retain their characteristic brightness and positions for about $100 \mathrm{~s}$. If this number were the same in other pulsars, carousel rotation times longer than $100 \mathrm{~s}$ could not be found by a periodicity search, as the characteristic traits of the subpulses will have changed before they return into view. For such long carousel rotation periods a different technique is needed.
In some pulsars there is a mechanism that can be used to circumvent the undersampling problem and hence determine the underlying carousel-speed: nulling. In these pulsars, a few percent of the pulses are missing as the pulsars suddenly turns off ("nulls"). After these nulls, the subpulse drift is often affected. Van Leeuwen et al. (2002, 2003) investigated this driftingnulling interaction in PSR B0809+74 and find it can only be explained if the underlying carousel rotates in over $200 \mathrm{~s}$, again much longer than expected. They predict that in pulsars with shorter carousel rotation times the drift direction should reverse after nulls.

In their 1983 paper, Lyne \& Ashworth mention the nullingdrifting interaction of PSR B0818-13 and show the post-null drift behaviour (their Fig. 14). For long nulls, there appears to be a reversed drift direction. Combining this with the prediction of van Leeuwen et al. (2003) we decided to further investigate the drifting-nulling interaction in PSR B0818-13.

\section{Observations and data reduction}

We have observed PSR B0818-13 from 2000 to 2003 with PuMa, the Pulsar Machine (Voûte et al. 2002) at the Westerbork Synthesis Radio Telescope (WSRT). The 11 observations amount to 20 hours in total or, using the $1.238 \mathrm{~s}$ period, $5.8 \times 10^{4}$ individual pulses. All observations were conducted at a time resolution of $0.4096 \mathrm{~ms}$ and around frequencies in between 328 and $382 \mathrm{MHz}$, with a bandwidth of several times $10 \mathrm{MHz}$, depending on interference. Each $10 \mathrm{MHz}$ band was split in 128 channels and dedispersed offline.

The data were searched for nulls and subpulses in the way described in van Leeuwen et al. (2002): for each observation 

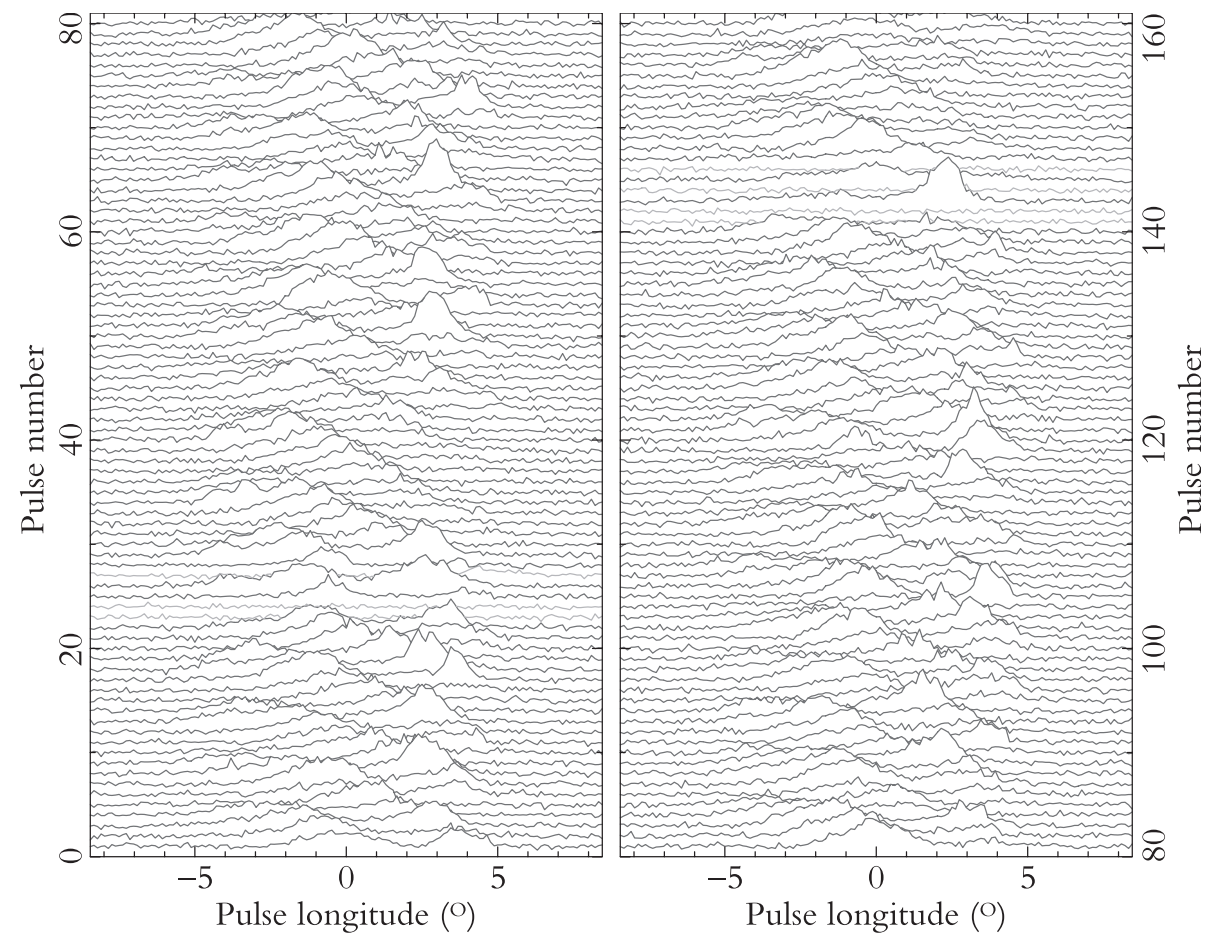

Fig. 1. Stacked single pulses with two intermittent nulls in lighter grey. we compose a histogram of the energy of individual pulses, like Fig. 2. The discrete population around zero-energy pulses is labeled "nulls". In each individual pulse, we fit Gaussian profiles to the subpulses. We determine which subpulse-fits are significant and store the subpulse positions, widths and heights along with the raw pulse data. As the subpulse-drift bands form regular structures, we use the predictive capability of the thus-found strong subpulses to detect weaker subpulses over the noise.

\section{Results: Analysis of nulls and subpulse drift}

\section{Statistics of short and long nulls}

PSR B0818-13 nulls about 1\% of the time. As the histogram of null lengths Fig. 3 shows, most of these nulls are very short, with lengths of only one or two periods. What the histogram doesn't show however, is that although roughly $2 / 3$ of these short nulls occur by themselves, many are observed in groups of intermittent pulses and nulls, like the groups seen in Fig. 1. As this behaviour is uncommon, Lyne \& Ashworth (1983) chose to disregard all intermittent short nulls, and only investigate longer nulls. But has the pulsar really turned off during such a long null? Or are the long nulls in PSR B0818-13 actually intermittent nulls where the series of short nulls happen to line up with our line of sight? This is the question we investigate.

If we assume that, during a train, the pulsar quickly and successively turns on and off, we can use the statistics of the null/pulse occurrence within a train to determine the likeliness ratio of null and pulse occurrence. To classify the different pulse trains, we have defined the first and last nulls of a train to be the start and end of the group. In Fig. 4 we give an overview of all event lengths. From this figure it is clear that the occurrence of null-groups as a whole is not governed by



Fig. 2. Energy histogram for PSR B0818-13. In light grey we show the energy distribution of the energies found in the on-pulse window. In black we show the energies found in an equally large off-pulse window, caused by system noise. The set of low-energy pulses detached from both the normal and the null distribution are probably half-nulls.

chance: there are too many long events. Within an event the pulsar blinks fairly quickly, but the change back to the normal pulse state usually takes several seconds.

The null group in the left panel of Fig. 1, for example, we classify as an event of length 5, while the one in the right panel is an event of length 6 . As the first and last nulls act as markers, there are only $6-2=4$ positions in the rightmost of Fig. 1 group that can be either nulls or pulses. In this case, there are two nulls and two pulses in this middle part, in a NNPNPN pattern. In Table 1 we give an overview of all the different nullpulse-trains present in our sample, in the same pattern notation. In the middle parts of all events, there are 238 possible 


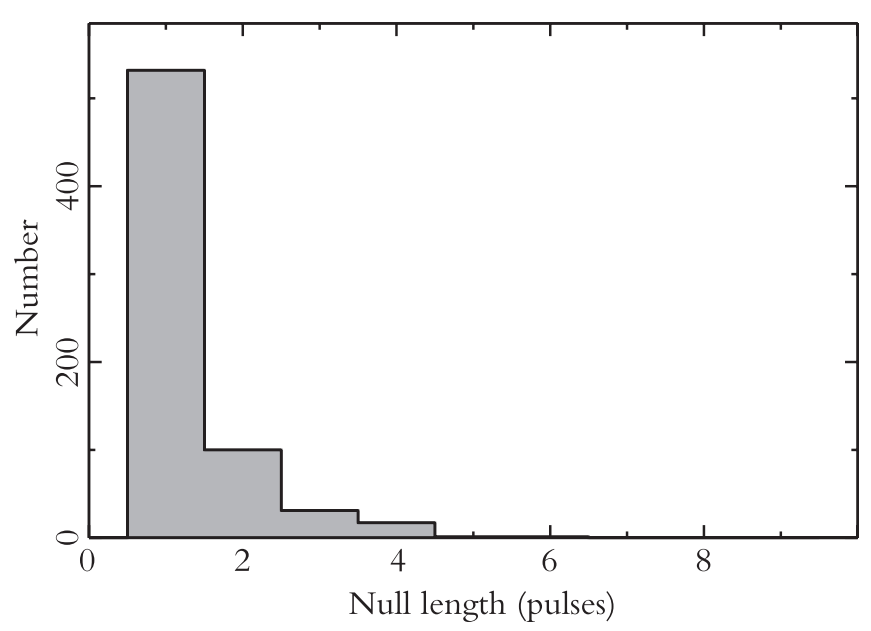

Fig. 3. Histogram of the null lengths.

locations to be divided between pulses and nulls. Of these, 107 are nulls, and 131 are pulses, so we conclude that, on average, pulses are 1.2 times more likely to occur within a group than nulls. Using this ratio, we can predict the chance that a train of a certain length appears to be one long null. If we consider that we probably miss or misidentify events that start or end with pulses in stead of nulls, the occurrence of long apparent nulls agrees quite well with the prediction. For groups of length 5, for example, the first and last positions are nulls by definition, but the middle 3 positions should be nulls, the probability of which is $\left(\frac{1.0}{1.0+1.2}\right)^{3}=0.09$. This is in good agreement with the observed ratio of 1 in 9 that follows from Table 1 .

This means that at the pulsar the intermittent pulse trains and the long nulls are the same. When the short nulls in such a train all happen to occur when the pulsar faces us, the effect only appears different.

We now know that during the null-trains, the pulsar blinks quickly, producing several nulls that are shorter than a pulse period. The continuous increase towards shorter nulls in nulllength histogram Fig. 3 already hinted at the existence of a large number of nulls shorter than 1 pulse period. Some of the starts and ends of these nulls must occur within the pulse window. Because of the variability normally already present in the pulse shapes, this dying or growing of the flux is not directly recognizable, but statistically it results in a lower energy for the pulse. In the histogram of pulse energies these "half-nulls", or "half-pulses" should be located in between the energy-values of the real nulls and the real pulses (the vertical lines in Fig. 2).

In the histogram, we do indeed see a number of these low-energy pulses (about $0.3 \%$, with energies varying from 10 to $40 \%$ of the average pulse energy). For a pulse to have $10-40 \%$ of the normal energy, the null should start (stop) in the last (first) $20 \%$ of the pulse window, so within $\pm 2^{\circ}$. If the positions of the starts and stops of null are random, we would expect to see this happen in $\frac{2}{360}=0.5 \%$ of pulses, in good agreement with the values found above. If these low-energy pulses are really half-nulls, we would expect them to occur preferentially near null trains, as normal nulls do. We therefore calculated the mean distance to a null for both normal and low-energy pulses. We find that low-energy pulses are on

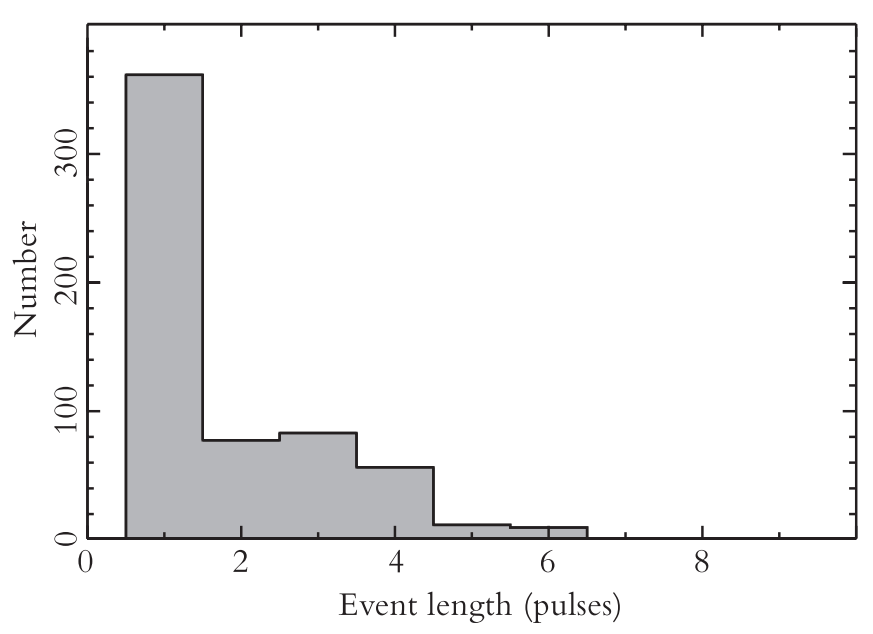

Fig. 4. Histogram of the event lengths.

Table 1. Length, pattern and occurrence of all null-groups in our sample. By definition, events always start and end with nulls. $\mathrm{N}$ denotes a null, $\mathrm{P}$ a pulse. Symmetric patterns are treated as one: NPNN for example is a combination of NPNN and NNPN. Within a group, observing a pulse is 1.2 times more likely than observing a null.

\begin{tabular}{cccccc}
\hline \hline $\begin{array}{c}\text { Event } \\
\text { length }\end{array}$ & $\begin{array}{c}\text { Pulse-null } \\
\text { combinations }\end{array}$ & $\begin{array}{c}\text { \# of } \\
\text { events }\end{array}$ & \# N & \# P & \\
\hline 1 & N & 361 & & & \\
2 & NN & 76 & & & \\
3 & NNN & 27 & 27 & 0 & 27 \\
& NPN & 52 & 0 & 52 & 52 \\
4 & NNNN & 16 & 32 & 0 & 32 \\
& NPPN & 13 & 0 & 26 & 26 \\
& NPNN & 19 & 19 & 19 & 38 \\
5 & NNNNN & 1 & 3 & 0 & 3 \\
& NPPPN & 2 & 0 & 6 & 6 \\
& NPPNN & 1 & 1 & 2 & 3 \\
& NPNPN & 1 & 1 & 2 & 3 \\
& NPNNN & 4 & 8 & 4 & 12 \\
6 & NNNNNN & 1 & 4 & 0 & 4 \\
& NPPPNN & 2 & 2 & 6 & 8 \\
& NPPNPN & 3 & 3 & 9 & 12 \\
& NPNPNN & 2 & 4 & 4 & 8 \\
& NPNNNN & 1 & 3 & 1 & 4 \\
\hline & Total: & & 107 & 131 & 238 \\
\hline & Fraction & & .45 & .55 & 1 \\
\hline
\end{tabular}

average about $30 \%$ closer to a null than normal pulses, so from this we conclude that at least some of the low-energy pulses are actually half-nulls, where we see the pulsar turning on or off while facing us.

\section{Profiles around nulls}

The next step in investigating how the pulsar turns on and off is to investigate changes in pulse type or shape before and 




Fig. 5. Average profile around nulls. Black line: average profile for normal pulses. Dotted line: average profile for all pulses directly before a null. Dashed line: average profile for all pulses directly after a null. We have used all (500+) nulls, including those in groups.

after nulls. First, we compare the number of subpulses in normal pulses with the number observed in pulses that lie in between nulls (cf. pulses 25, 26, 143 and 145 in Fig. 1). We find that on average the former contain $1.79 \pm 0.01$ subpulses, the latter $1.80 \pm 0.05$. To compare pulse shapes, we have averaged the last and first active pulses around all nulls in our sample, as shown in Fig. 5.

The profile of the first pulse after the null is roughly as expected; as the pulsar sometimes turns back on only halfway through the pulse window, we expect the leading edge of the profile to diminish compared to the trailing edge, and this we indeed see. For the last pulse before the null one would expect the opposite (the leading edge should become relatively more important as the pulsar sometimes already turns off near the end of the null), but this we do not see. The last pulse before the null actually shows a stronger trailing part, contrary to what we would expect.

Finally we note that the first and last active pulse more clearly show two-peaked profiles than the average profile does. As the separation between the peaks is roughly equal to the subpulse separation $\mathrm{P}_{2}$, they could be explained by the pulsar turning on and off at certain preferred subpulse-carousel positions. In this case the subpulses would not smear out in the average, as in the normal profile, but add up. We have not been able to verify this suggestion in an independent way however, so we put it forward with some reservation.

\section{Profiles around low-energy pulses}

As some of the low-energy pulses are probably partial nulls, we also investigated the profiles of these pulses and the pulses surrounding them. Similarities between the profiles around nulls and low-energy pulses, would again suggest they are related. We averaged the profiles in and around 80 low-energy pulses, as shown in in Fig. 6.

First thing to note is the two-peaked profile of the average low-energy pulses themselves, with the peak separation equal to the subpulse separation. This indicates that certain subpulsepositions are overrepresented, a conclusion strengthened by the

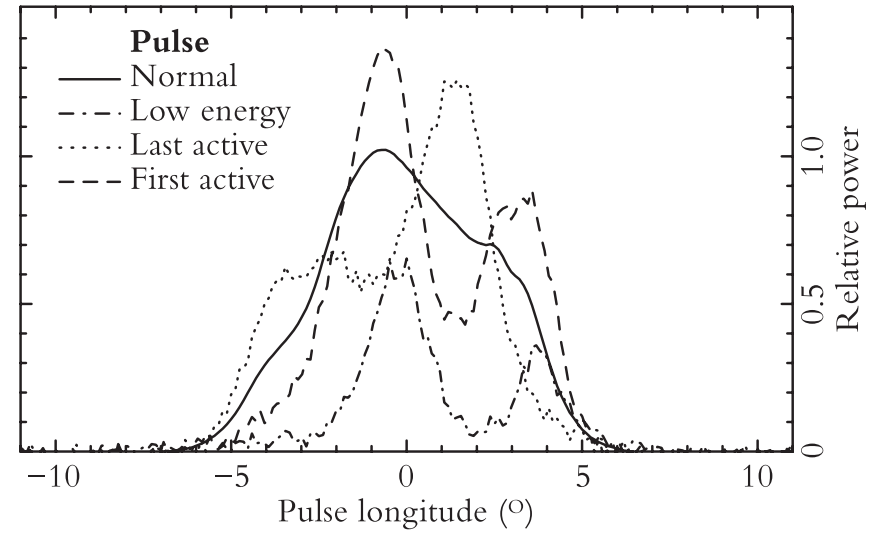

Fig. 6. Average profile around lows, as in Fig. 5. Again, the black line represents the average pulse profile. The dotted and dashed lines are the profiles before and after the low in this case. The dashed-dotted line shows the average profile for the low-energy pulses.

fact that the last and first active pulses are offset by the same amount as would be expected based on normal subpulse drift. Apparently, normal pulses with certain subpulse positions are less bright than average and contaminate the equally dim halfnull sample.

\section{Position jumps over nulls}

Looking at the subpulse drift in PSR B0818-13 in Fig. 1, the drift direction seems obvious (slowly towards earlier arrival) but in reality it might be different. The subpulses lack clear, recognizable traits (width, height or spacing) so instead of moving slowly towards earlier arrival, the subpulses might actually move more quickly towards later arrival, and still produce the same image as Fig. 1. As we only observe the subpulse position once per pulse period, we undersample the subpulse movement and cannot determine the underlying speeds immediately (see also Fig. 2 in van Leeuwen et al. 2003).

By focusing on drift behaviour around nulls, we hope to obtain more information about the subpulse-drift alias mode and the rotation speed of the underlying carousel, like we previously did for PSR B0809+74 (van Leeuwen et al. 2003). For each null in our sample, we determine the positions of the preceding and following subpulses and calculate the phase jump of the subpulse system over each null.

In Fig. 7a we plot the longitude jump of the subpulse system versus null length, for all nulls in our sample. As the subpulses are indistinguishable, it is not clear which pre-null subpulse corresponds to which post-null subpulse. Therefore, in Fig. 7a, all the data is mapped into one region that is $\mathrm{P}_{2}$ (the average subpulse separation) wide. To visualize the data better, we have recreated the subpulse pattern by duplicating the data-points with offsets of $\mathrm{N} \times \mathrm{P}_{2}$ in Fig. 7b. The real grouping of the subpulses is now much more apparent. This diagram is analogous to Fig. 1, but is now thought to show the drifting of the subpulses during the null, when they are not actually visible. We have also added some simulated pre-null subpulse positions in the lower half of the diagram, for clarity. 


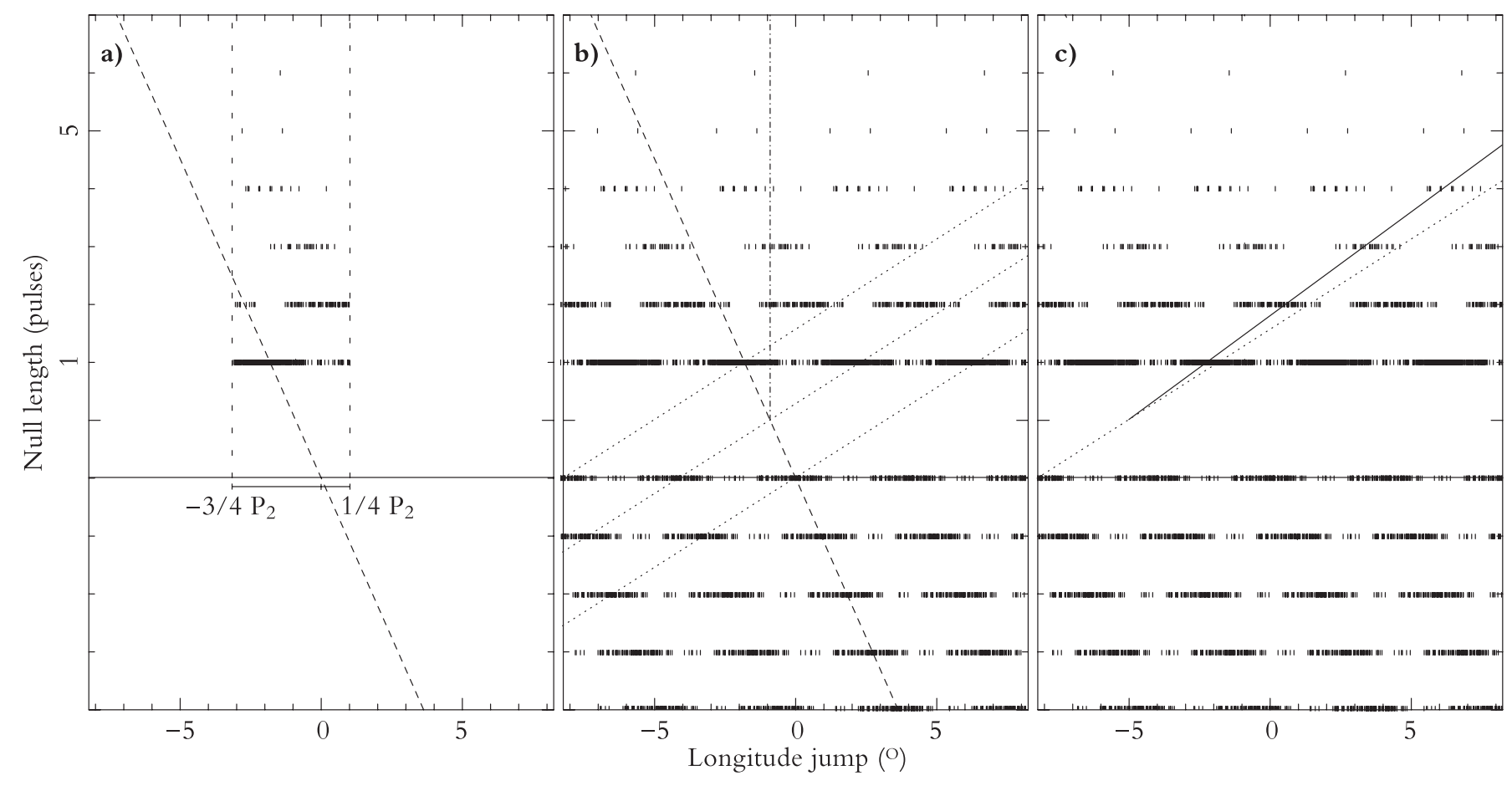

Fig. 7. Subpulse-pattern longitude jumps over continuous nulls. Each mark is the jump over one null. a) Mapped into a region from $-\frac{3}{4} \mathrm{P}_{2}$ to $\frac{1}{4} \mathrm{P}_{2}$ around the expected location in the continuous-drift case. b) The same data, duplicated to show subpulse grouping, with simulated pre-null subpulse positions added for clarity. The vertical dashed-dotted line predicts subpulse positions for a total stop of all drift during the null. The dashed lines are the prediction if drifting continuous during the null as it did before, for non-aliased drift. The dotted lines show which subpulses one spark makes if the drift is aliased. c) The same data, now with the predicted aliased subpulse path (dotted line), and the actual lower driftrate (solid line).

If, during the null, the subpulse would stop drifting, the subpulses should follow the vertical dashed-dotted line in Fig. 7b. If the subpulses continue to drift during the null, they should follow the dashed line. Neither of the two is a particularly good fit. After the null, the subpulses appear to outrun the predictionline, which would indicate the drift-speed has increased during the null.

\section{The subpulse drift alias mode}

For the dashed line in Fig. $7 \mathrm{~b}$ we have assumed that the drifting is not aliased, i.e. that one driftband is made by only one spark. Another option would be that the carousel is rotating the other way (as in Fig. 2b in van Leeuwen et al. 2003). In this case, a single spark does not move towards earlier arrival and follow the dashed line in Fig. 7b. It moves towards later arrival and follows one of the dotted lines.

Compared to these dotted lines of expected positions, the post-null subpulses are lagging, which means the drifting slows down during a null. This would be consistent with the behaviour of the only other well-studied case of drifting-nulling interaction, in PSR B0809+74. There the spark-carousel unambiguously decelerates during a null, even to the point of a total stop. If we assume that our two-pulsar sample behaves similarly instead of oppositely, the spark carousels in both should slow down during nulls, and therefore the real drift direction in PSR B0818-13 must be towards later arrival. It could do so as illustrated by the dotted lines in Fig. 7b, but faster too. If we assume the carousel consists of 20 sparks, similar to PSR B0943+10 Deshpande \& Rankin (1999) and PSR B0809+74 (van Leeuwen et al. 2003), we can place an upper limit on the carousel rotation time. In the slowest aliased case, a subpulse takes 1.3 pulse periods $P$ to reach the position of its neighbour (i.e. the vertical spacing of the dotted lines in Fig. 7b). The whole carousel then rotates in $1.3 \times P \times N_{\text {sparks }} \sim 30 \mathrm{~s}$. This already makes PSR B0818-13 the pulsar with the fastest carousel rotation time known; if the subpulse drift is more strongly aliased, the rotation time is even smaller.

\section{Subpulse drift during intermittent nulls}

If the carousel in PSR B0818-13 slows down during a null, then why doesn't it stop like the carousel in PSR B0809+74 does? Again, the answer is in the intermittent nature of the apparent long nulls. What appears to be a long null is actually a series of short nulls: during each of these the carousel may slow down, only to speed up again in between. On average, this comes down to a steady lower carousel speed during nulls. In Fig. 7, this would translate to a straight line through the post-null subpulse groups, and this is in good agreement with the data, as the full line in Fig. 7c shows. If we assume the alias mode as illustrated by the dotted line in Fig. 7c, the average driftrate (full line) during a long apparent null is about $0.87 \pm 0.05$ of the normal driftrate (dotted line). Using the pulse/null ratio of 1.2 we found previously, this means that 


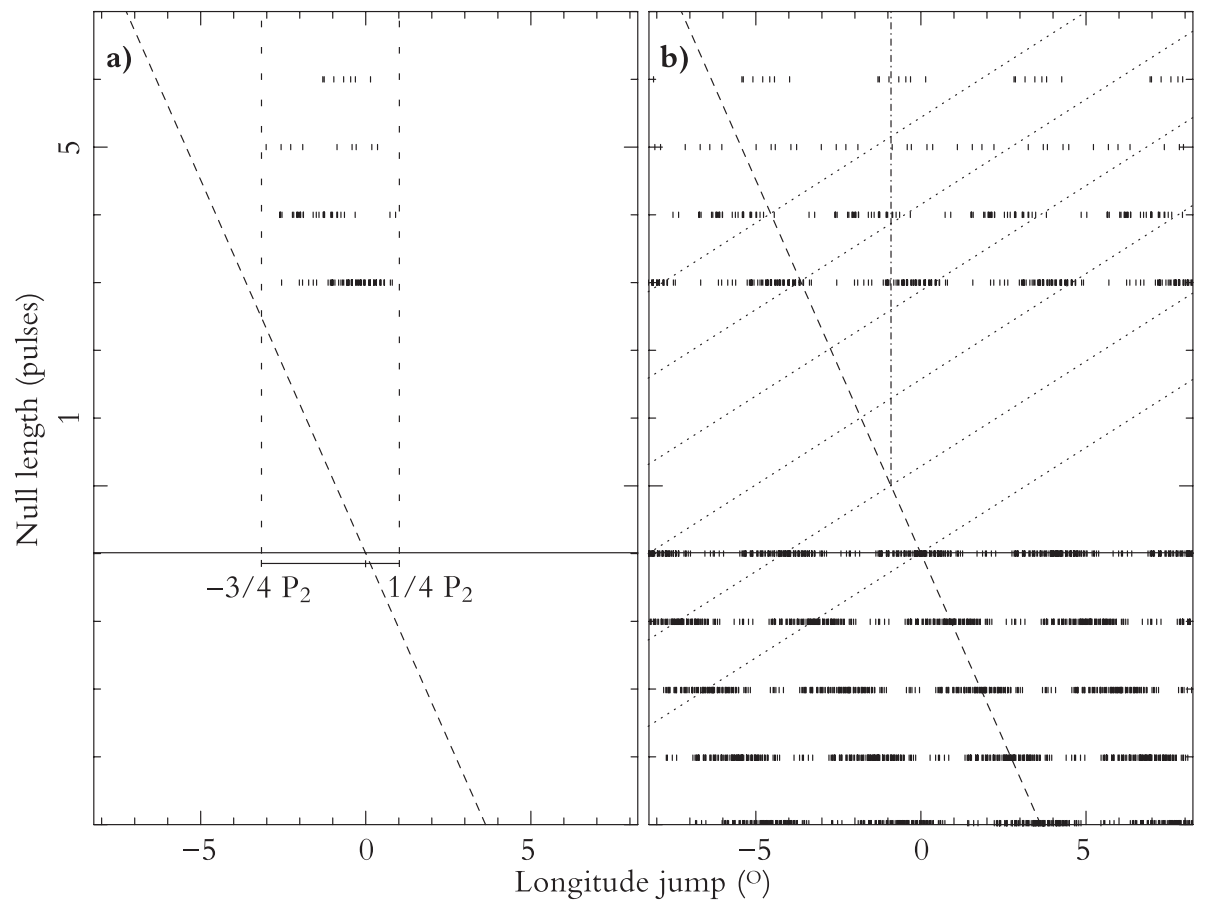

Fig. 8. As Fig. 7, but now for intermittent nulls. during the short nulls that compose this long apparent null, the drift must be less than half the normal driftspeed to produce the apparent driftspeed observed.

If this is correct, and the slow drift-rate seen in the apparent long nulls is due to the subpulse drift starting and stopping every time a short null changes into a short burst, we must find the same lower driftrate over the intermittent null-groups. In Fig. 8 we show the subpulse pattern over these intermittent nulls in the same fashion as Fig. 8 did for continuous nulls. Again we note that the subpulses arrive earlier than expected. The similarity in Figs. 7 and 8 is striking; even in their drift-rate, the continuous and intermittent nulls behave identically.

\section{Discussion}

In a rotating-carousel model, the groups of subsequent short nulls and pulses can be explained in two ways. In the one we have thus far focussed on, the entire carousel quickly stops and starts emitting. In this model all subbeams pause at the same time. In a different model, only some subbeams in a carousel stop emitting while the carousel rotation continues, creating pulse-null patterns similar to those observed. When the off-part of the carousel rotates into view, one observes a null. One pulse period later, an on-part might have moved into the pulse window to produce a normal pulse. Such a configuration would have to meet some very specific criteria, though. If the pattern is caused by on and off-parts of the carousel turning in and out of view, one should also observe half-on half-off pulses, with fewer subbeams. Yet we have found that the pulses in pulsenull groups contain exactly as many subpulses as normal pulses do. Only a carousel with many subbeams (roughly several hundreds) at a very high rotation speed could begin to explain this lack of observed on- to off-part transitions, but with a subpulse separation of $4^{\circ}$, the inferred size of the total carousel appears unrealistic.
When we compare pulsars B0809+74 and B0818-13, the similarities are striking. Both shine normally for $98-99 \%$ of the time, to be occasionally interrupted for several seconds and then resume their normal emission. Both show similar changes in driftrate during nulls. It seems clear we see the same mechanism at work in these two pulsars. Yet for PSR B0809+74 we know the long nulls must be real, as the subpulse drift stops during these nulls (van Leeuwen et al. 2003), while in PSR B0818-13 we never see long nulls. There we observe periods of intermittent emission and quietude, in groups about as long as the nulls in PSR B0809+74.

Apparently, the mechanism that causes the emission in PSR B0809+74 to fully turn off, only reaches a border-line state in PSR B0818-13. Why the chances for null or pulse occurrence within such a state should be almost equal is unclear. The long timescales involved (even in the intermittent nulls) remains the biggest problem. In a polar-gap model (the basic physics of which are already debatable) for example, the potential gap over the magnetic pole can build up or discharge in microseconds (Ruderman \& Sutherland 1975). Why the pulsar would blink on a timescale of seconds, for $10 \mathrm{~s}$ in total, as it does in PSR B0818-13 remains unclear; we have not been able to explain this behaviour with any current pulsar theory. What one can do, is study the behaviour of different pulsars to find underlying patterns, which is what we have now done for PSR B0809+74 and PSR B0818-13.

The carousel rotation times found in PSR B0943+10 (41 s) and PSR B0809+74 (>200 s) pose a similar time-scale problem; in a simple polar-gap theory, the rotation times are roughly several seconds (Ruderman \& Sutherland 1975) and the rotation times found thus-far are much larger. The upper limit of $30 \mathrm{~s}$ we find for PSR B0818-13 extends the sample, back towards the previously expected values. A theory like the abovementioned also predicts that the rotation times scale with pulse period $P$ and magnetic field strength $B$ as $\frac{B}{P^{2}}$, which implies 
that the rotation time of PSR B0818-13 should be 3 times longer than that of PSR B0809+74, instead of the more than 10 times shorter we find. Our determination of the carousel rotation time in PSR B0818-13 is therefore a step to finding the dependencies of carousel rotation times on other pulsar parameters empirically.

\section{Conclusions}

Both the apparently long nulls and the groups of intermittent nulls can be quantitatively explained by trains of short nulls and pulses. The subpulse-drift behaviour over the two is identical: during the nulls, the subpulse drift appears to speed up. Assuming the underlying sparks slow down, as they do in PSR B0809+74, we conclude that the subpulse drift in PSR B0818-13 is aliased. This then leads to a carouselrotation time of less than $30 \mathrm{~s}$, the fastest one found to date.

\section{References}

Deshpande, A. A., \& Rankin, J. M. 1999, ApJ, 524, 1008 van Leeuwen, A. G. J., Kouwenhoven, M. L. A., Ramachandran, R., Rankin, J. M., \& Stappers, B. W. 2002, A\&A, 387, 169

van Leeuwen, A. G. J., Stappers, B. W., Ramachandran, R., \& Rankin, J. M. 2003, A\&A, 399, 223

Lyne, A. G., \& Ashworth, M. 1983, MNRAS, 204, 519

Ruderman, M. A., \& Sutherland, P. G. 1975, ApJ, 196, 51

Voûte, J. L. L., Kouwenhoven, M. L. A., van Haren, P. C., et al. 2002, A\&A, 385, 733

Wright, G. A. E. 2003, MNRAS, 344, 1041 